\title{
Application of indocyanine green video angiography in parasagittal meningioma surgery
}

\author{
Alessandro Della Puppa, M.D., ${ }^{1}$ Oriela Rustemi, M.D., ${ }^{1}$ Giorgio Gioffrè, M.D., ${ }^{1}$ \\ Giuseppe Rolma, M.D., ${ }^{2}$ Marzia Grandis, M.D. ${ }^{3}$ Marina Munari, M.D., ${ }^{3}$ \\ and Renato Scienza, M.D. ${ }^{1}$ \\ ${ }^{1}$ Department of Neurosurgery, Padua University Hospital; ${ }^{2}$ Neuroradiology Unit, Padua University \\ Hospital, Padua; and ${ }^{3}$ Department of Anaesthesiology, University of Padua, Italy
}

\begin{abstract}
Object. There are no doubts about the role that indocyanine green video angiography (ICGVA) can play in current vascular neurosurgery. Conversely, in brain tumor surgery, and particularly in meningioma surgery, this role is still unclear. Vein management is pivotal for approaching parasagittal meningiomas, because venous preservation is strictly connected to both extent of resection and clinical outcome. The authors present the technical traits and the postoperative outcome of the application of ICGVA in patients undergoing parasagittal meningioma surgery.

Methods. The authors retrospectively collected demographic, radiological, intraoperative, and follow-up data in 43 patients with parasagittal meningiomas who underwent surgery with the assistance of ICGVA at Padua Neurosurgical Department between October 2010 and July 2013. Intraoperative ICGVA findings at different stages (before dural opening, after dural opening, during resection, after resection) were reviewed. Additional data on functional monitoring, temporary venous clipping, and flow measurements were also recorded. The overall postoperative outcome was evaluated by assessing both the extent of resection and the clinical outcome data.

Results. The ICGVA studies were performed 125 times in 43 patients, providing helpful data for vein management and tumor resection in all stages of surgery. In $16 \%$ of meningiomas completely occluding the superior sagittal sinus, the ICGVA data differed from radiological findings and changed the surgical approach. In $20 \%$ of cases the intraoperative ICGVA findings directly guided the surgical strategy: venous sacrifice was necessary in 7 cases, without postoperative consequences; temporary clipping with neurophysiological monitoring proved to be predictive of safe venous sacrifice. In $7 \%$ of cases the ICGVA data needed to be supplemented with flow measurements. Simpson Grade I-II and Grade III resections were achieved in $86 \%$ and $14 \%$ of cases, respectively, with a $4.6 \%$ rate of overall morbidity.

Conclusions. This study shows that ICGVA can assist the different stages of parasagittal meningiomas surgery, guiding the vein management and tumor resection strategies with a favorable final clinical outcome. However, in the authors' experience the use of other complementary tools was mandatory in selected cases to preserve functional areas. Further studies are needed to confirm that the application of ICGVA in parasagittal meningioma surgery may improve the morbidity rate, as reported in this study.
\end{abstract}

(http://thejns.org/doi/abs/10.3171/2013.12.FOCUS13385)

KEY WORDS $\bullet$ parasagittal meningioma $\bullet \quad$ indocyanine green fluorescence
video angiography $\bullet \quad$ temporary venous clipping $\bullet$ meningioma treatment
venous flow measurement $\quad \bullet \quad$ functional monitoring

$\mathrm{P}$ ARASAgitTal meningiomas account for $20 \%-30 \%$ of all intracranial meningiomas. ${ }^{1}$ To date, whether the appropriate strategy for resection of parasagittal meningiomas should entail an aggressive approach aiming at complete resection ${ }^{19}$ rather than leaning toward a main purpose of venous and function preservation is still under debate..$^{22,23}$ A recent MEDLINE review of the literature on parasagittal meningiomas published over the last 60 years reported no evidence that aggressive manage-

Abbreviations used in this paper: DSA = digital subtraction angiography; ICGVA = indocyanine green video angiography; MEP = motor evoked potential; SSEP = somatosensory evoked potential; SSS $=$ superior sagittal sinus. ment presents an advantage in terms of recurrence rate. ${ }^{23}$ More particularly, the extent of resection of WHO Grade I meningiomas does not predict both recurrence-free survival rate ${ }^{20}$ and the tumor control rate, even with a close follow-up..$^{21}$ With regard to the specific topic of superior sagittal sinus (SSS) invasion, it has been reported that a small coagulated intrasinusal remnant (Simpson Grade III resection) tends to remain stable over time, ${ }^{22,23}$ thus making acceptable the cost of leaving a small remnant within a partially occluded sinus for a safer venous outflow. ${ }^{6}$

The consequence of these emerging data is a surgical strategy with function preservation as its first purpose. The venous preservation-guided resection ${ }^{23}$ seems 


\section{A. Della Puppa et al.}

consequently to dominate the latest case series, in contrast to the more radical resections and vein reconstruction advocated by previous studies. ${ }^{19}$ There is no debate that vein preservation is the best policy for the postoperative functional outcome, as far as the risk of potential venous damage is concerned. Injury to cortical and bridging veins during surgery has been associated with poor outcomes; therefore, its avoidance has been strongly advocated..$^{8,11,15,17}$ Indocyanine green video angiography (ICGVA) is an intraoperative tool that can provide anatomical and functional data on venous flow. In the present study, we report our experience in 43 patients affected by parasagittal meningioma who underwent surgery in our institution with the assistance of ICGVA. Our strategy was to achieve maximal resection with function preservation, by using a vein management protocol guided by the intraoperative ICGVA data. The purpose of the study was to critically review our data on the intraoperative use of ICGVA, to describe the intraoperative technical nuances, and to eventually assess the outcome of this strategy.

\section{Methods}

We retrospectively collected demographic, radiological, intraoperative, and follow-up data in 43 consecutive patients with parasagittal meningiomas who underwent resection with the aid of ICGVA at our department between October 2010 and May 2013. The enrollment criteria were as follows: diagnosis of parasagittal meningioma, and surgery assisted by ICGVA. In all cases an MRI study was obtained with Gd contrast before surgery. Sinus involvement and collateral venous pathways were assessed in all cases by using digital subtraction angiography (DSA) preoperatively with late venous phases and/ or MR angiography studies. The patients were stratified according to the lesion size (meningiomas were considered large when their major diameter was $\geq 3 \mathrm{~cm}$ and small if their diameter was $<3 \mathrm{~cm}$ ), location (anterior, middle, or posterior third of SSS), and degree of sinus invasion according to the Sindou classification. ${ }^{17-19}$

\section{Application of ICGVA and Surgical Strategy}

Our strategy entailed the achievement of maximal resection with function preservation, through venous management based on intraoperative ICGVA data. The ICGVA was performed at different stages during surgery, either alone or, in selected cases, in combination with different tools in a multimodal strategy. More specifically, ICGVA was performed as follows.

1) Before dural opening, ICGVA (ICGVA I) was performed to achieve a transdural assessment of the tumor site and cortical veins for a tailored and safe dural opening (Fig. 1). When sinus occlusion was suspected preoperatively, the sinus roof was completely exposed to confirm sinus occlusion with ICGVA. If there was evidence of sinus occlusion on ICGVA (Fig. 2), an en bloc resection of the meningioma and the involved sinus was performed according to intraoperative ICGVA data. Conversely, when the preoperative findings did not suggest a sinus occlusion, the sinus roof was only partially exposed.

2) After dural opening, ICGVA (ICGVA II) was per- formed to assess the venous anatomy, the relation of the veins with the tumor, the direction of venous flow (anterograde or retrograde), and the functional status of the veins (functioning or thrombosed veins) (Fig. 3).

3) An ICGVA study was additionally performed during resection, when needed (ICGVA III). In cases in which venous sacrifice was necessary, ICGVA was always performed to evaluate the existence of collateral circles, after temporary clipping of the vein to be sacrificed (Fig. 4).

4) At the final stage, after resection of meningioma, ICGVA (ICGVA IV) was performed to evaluate the final venous pattern and to visualize possible venous functional changes (Fig. 3).

A multimodal strategy was adopted in selected cases, depending on the preoperative radiological features and intraoperative findings. Intraoperative neurophysiological monitoring, somatosensory evoked potentials (SSEPs), and motor evoked potentials (MEPs) as well as functional mapping were performed according to both the size and location of tumor and the preoperative pattern of SSS invasion. Cortical and subcortical mapping assisted meningioma resection by identifying motor areas to safely respect the cleavage plane between tumor and mapped areas. Temporary venous clipping (Fig. 4) was performed only when venous sacrifice was deemed necessary to improve resection and when neurophysiological monitoring was available. The ICGVA data represented the first step toward identifying which tract of the vein could be safely occluded by the visualization of a collateral circulation. For this purpose, a temporary clip was positioned during ICGVA. If a collateral circulation was not present, the temporary clip was removed. Conversely, if a collateral circulation was present (Fig. 4c), MEP and SSEP amplitude was monitored for a few minutes; if no changes were recorded, the vein was definitively occluded at the exact point of the temporary clip previously positioned. Venous flow measurements were additionally performed using a microvascular ultrasonic flow probe (Charbel Microflowprobe; Transonic Systems, Inc.) ${ }^{1}$ (Figs. 3 and 4) when the flow data provided by ICGVA were not satisfactory. With regard to the pattern of sinus invasion, if the meningioma was simply attached to the outer surface of the sinus wall (Type I), the target was a complete resection. If any intrasinusal invasion was detected (Types II, III, and IV), the tumor was resected and the sinus wall repaired either with sutures, glue and muscle, or Gelfoam. When sinus occlusion was intraoperatively confirmed by ICGVA (Type V-VI), sinus ligation and section were carried out, and the meningioma was removed en bloc.

\section{Assessment of Postoperative Outcome}

The postoperative outcome was evaluated according to the postoperative morbidity and tumor resection rate. Morbidity was defined by the evaluation of new neurological deficits and/or neurosurgical complications. Additionally, the functional status was measured by the Functional Independence and Mobility Score, which allows a stratification of the patients on the basis of feeding abilities, locomotion, expression, transfer mobility, and social interaction. Depending on this score, patients were grouped into one of the following categories of independence: 1 (complete dependence); 2 (modified dependence); 3 (modified inde- 

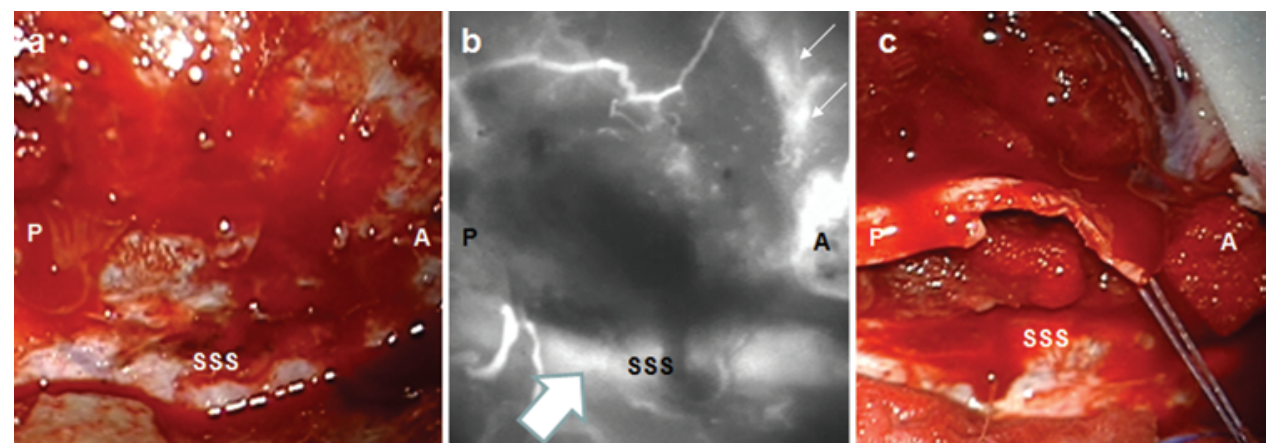

FIG. 1. Intraoperative views showing a parasagittal meningioma of the middle third (nonoccluding) of the SSS in a 62-year-old woman. a: The ICGVA study is performed before dural opening (ICGVA I). b: An ICGVA study showing SSS location and patency (large white arrow) and cortical vein location (small white arrows), guiding resection. c: Intraoperative photograph showing a safe dural opening. $A=$ anterior; $P=$ posterior.

pendence); and 4 (complete independence). ${ }^{10}$ The extent of resection was stratified using the Simpson grading scale. ${ }^{16}$ According to a strict interpretation of the Simpson grading system, Grade III resection (gross total) describes a type of resection in which tumor tissue is left within the SSS. ${ }^{16}$ There has been some confusion regarding the intrasinusal remnant and its collocation in Simpson grading; ${ }^{7}$ we have adopted the term Grade III resection for the small intrasinusal remnants, considering their relatively low recurrence rate (approximately $8 \%$ ), especially for WHO Grade I meningiomas. ${ }^{20,22,23}$

\section{Results}

\section{Patient Population}

Data are summarized in Table 1. The enrolled patients were 28 women and 15 men. The median age was 65 years (range 37-81 years). Thirty-seven patients were affected by a newly diagnosed and 6 by a recurrent meningioma. The tumor localization was as follows: 11 patients $(25 \%)$ had meningiomas located in the anterior third; 19 (44\%) had meningiomas located in the middle third; and $13(31 \%)$ had meningiomas located in the posterior third of the SSS. As far as the tumor size is concerned, 25 patients $(58 \%)$ had large meningiomas and $18(42 \%)$ had small meningiomas. Twelve patients had radiological evidence of SSS occlusion, whereas in the other 31 patients the SSS was still patent. According to the Sindou classification ${ }^{17-19}$ of sagittal sinus involvement, $22(51 \%)$ of 43 meningiomas were classified as Type I, 7 (16\%) were Type II-III, 4 (9\%) were Grade IV, and 10 (24\%) were Type V-VI. Meningiomas were WHO Grade I in 37 patients and Grade II in 6 patients.

\section{Strategy for ICGVA-Guided Resection}

The ICGVA studies were performed 125 times (Fig. 5); ICG was injected in all cases at least twice (in the intradural stage and before and after tumor resection). An ICGVA I study (before dural opening) was performed 29 times, and in $85 \%$ of cases, it allowed the visualization of
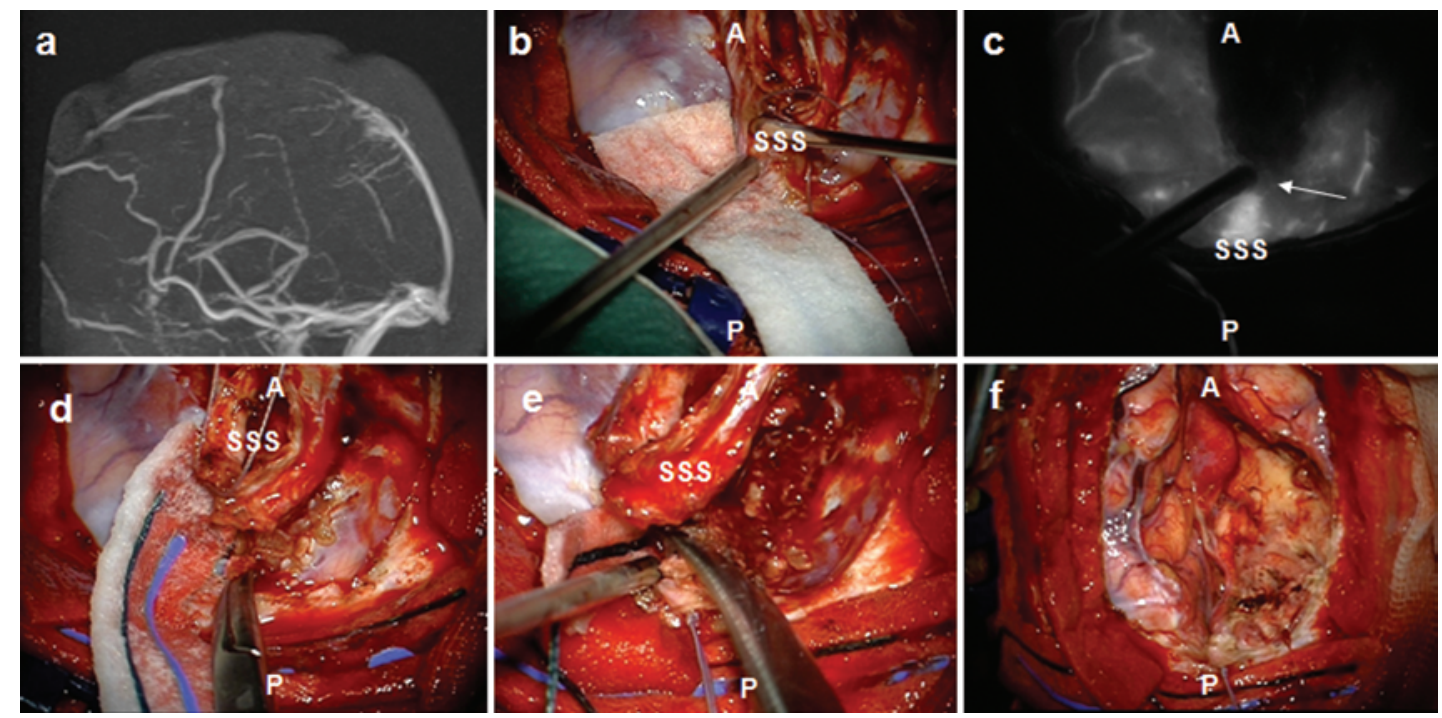

FIG. 2. a: An MR angiography study obtained in a 51-year-old man with parasagittal meningioma of the middle third (completely occluding) of the SSS. b-f: Intraoperative views. The posterior limit of the occluded SSS (b). An ICGVA study showing the point of filling interruption (c, white arrow), guiding surgery. Surgical ligation of the SSS (d). Section of the SSS (e). Meningioma resection (f). 

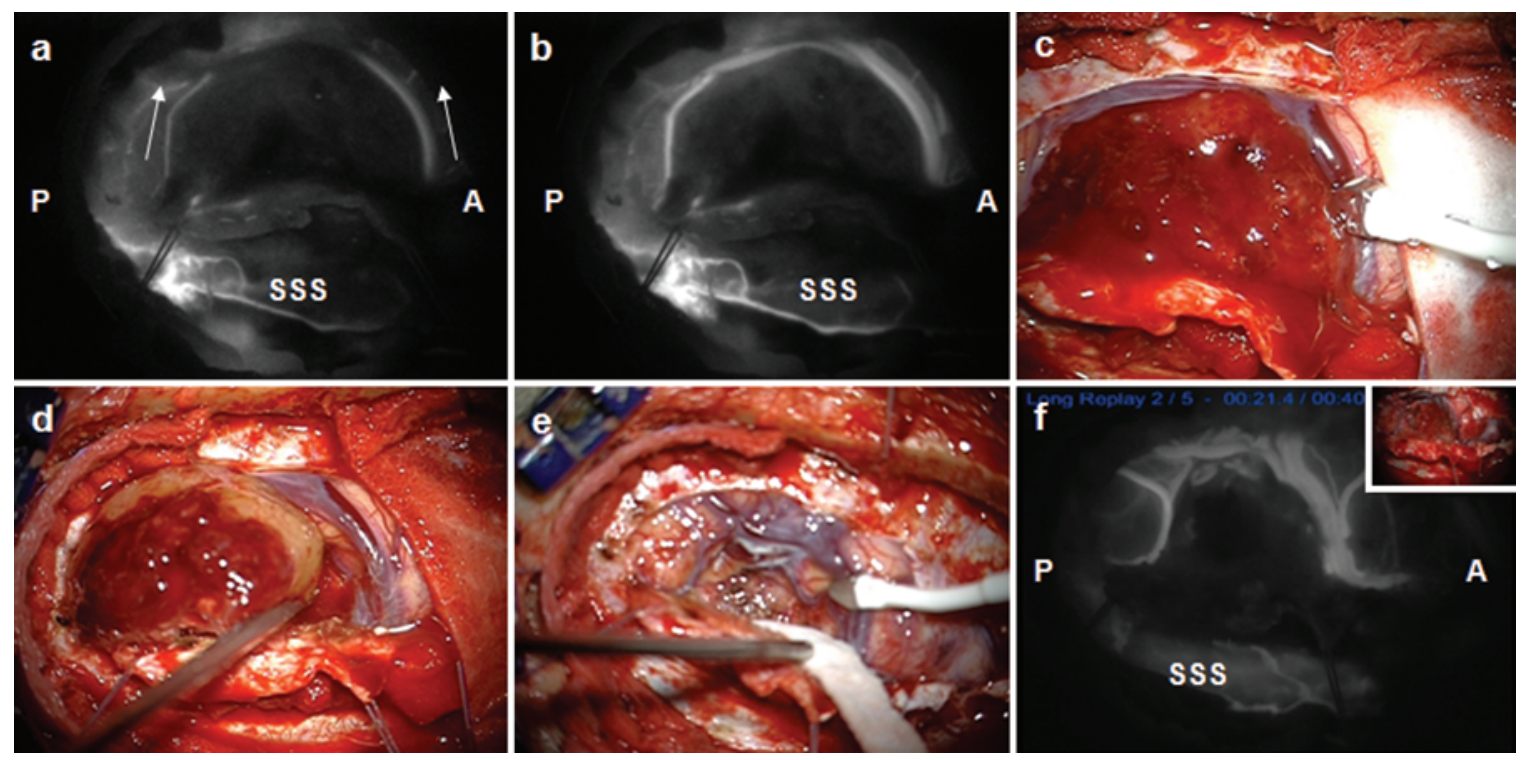

FIG. 3. Views obtained in a 71-year-old woman with parasagittal meningioma of the middle third, not occluding the SSS. The ICGVA is performed before tumor resection (ICGVA II). The ICGVA studies (a and $\mathbf{b}$ ) show cortical vein distribution around meningioma, with evidence of retrograde venous flow (arrows). Flow measurement by microflow probe (c) allows a repeat monitoring during tumor resection (d and e). Final view (ICGVA IV) showing vein preservation (inset) and increased flow in comparison with the preresection status (f).

the meningioma: boundaries of tumor were clearly detectable, and the tumor itself showed a heterogeneous pattern of ICG enhancement. Cortical veins could often be identified at this stage and guided a tailored dural opening (Fig. 1). Among the 12 cases in which the radiological findings suggested sinus occlusion, in 10 cases such an occlusion was confirmed (Fig. 2), and in 2 cases the dye slowly filled the sinus (Fig. 6). After dural opening, ICGVA was performed in all cases before (ICGVA II) and at the end of resection (ICGVA IV). In 10 cases an additional ICG injection was required during tumor removal (ICGVA III): in 7 of those cases venous sacrifice was performed ( 3 anteriorthird, 3 middle-third, and 1 posterior-third meningioma), in all cases for large tumors. In 3 of 7 cases the veins appeared to be thrombosed at ICGVA; the vein was sectioned and followed by ICGVA reassessment. In 4 of 6 cases the veins were patent and required temporary clipping.

Intraoperative neurophysiological monitoring of SSEPs and MEPs was performed in 12 patients-10 (55\%) of the 19 who had meningiomas in the middle third of the SSS, and 2 of the 13 who had posterior-third meningiomas (15.4\% of all posterior-third meningiomas). Neurophysiological monitoring was used in 6 of the 10 patients with radiological evidence of sinus occlusion and in 6 of the 33 patients without such evidence. More particularly, in 12 patients continuous MEP and SSEP monitoring was used during tumor resection. In 5 cases functional mapping was performed, and in 3 cases the motor area was mapped. Temporary clipping was performed in 5 cases, always under neurophysiological monitoring, and in all 5
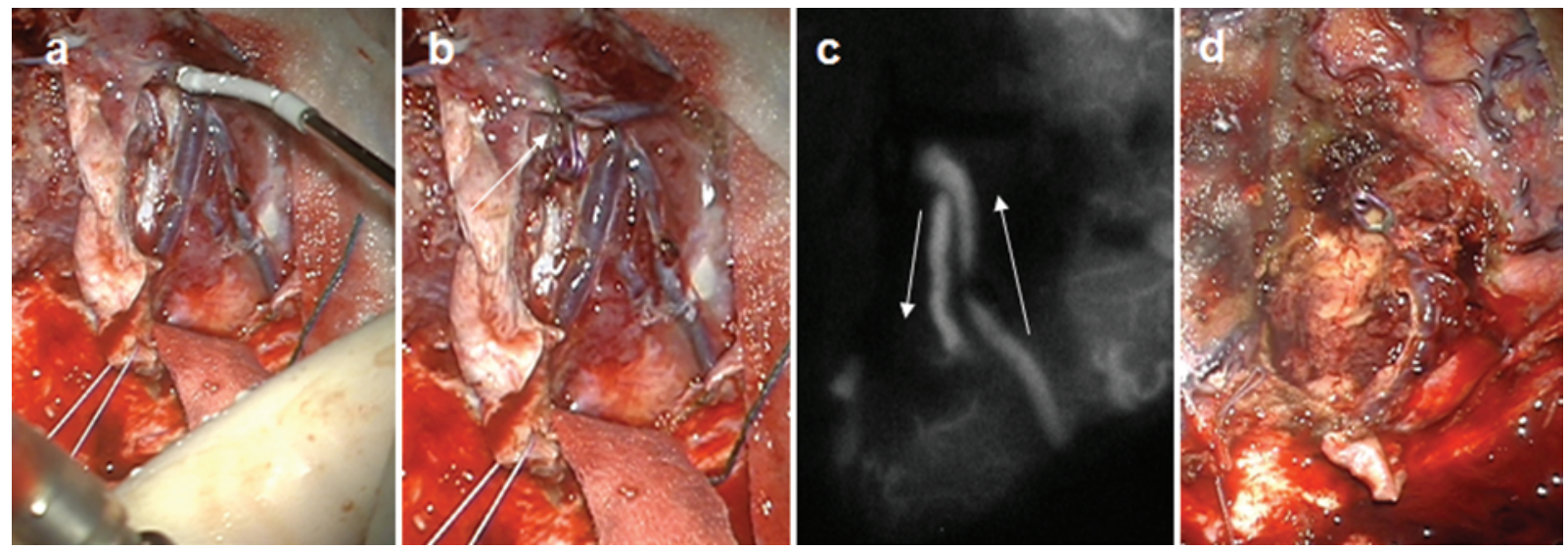

FIG. 4. Views obtained in a 57-year-old man with parasagittal meningioma of the middle third, not occluding the SSS. Intraoperative views: a bridging vein runs just upon the meningioma. The complex distribution of veins around the meningioma prompts an additional flow measurement (a) of all veins before temporary clipping (b) (white arrow) under neurophysiological monitoring. ICGVA (III) injection shows a collateral flow (arrows, c) without changes of evoked potentials amplitude; thus, venous sacrifice is performed and complete tumor resection is achieved (d). 
The ICGVA-guided resection of parasagittal meningiomas

TABLE 1: Features and surgical outcome in 43 patients with parasagittal meningiomas

\begin{tabular}{|c|c|}
\hline Parameter & Value (\%) \\
\hline no. of patients & 43 \\
\hline median age in yrs & 65 \\
\hline range & $37-81$ \\
\hline $\operatorname{sex}(M / F)$ & $15: 28$ \\
\hline \multicolumn{2}{|l|}{ tumor status } \\
\hline newly diagnosed & $37(86)$ \\
\hline recurrent & $6(14)$ \\
\hline \multicolumn{2}{|l|}{ WHO grade } \\
\hline I & $37(86)$ \\
\hline II & $6(14)$ \\
\hline III & $0(0)$ \\
\hline \multicolumn{2}{|l|}{ tumor characteristics } \\
\hline large; $\geq 3 \mathrm{~cm}$ & $25(58)$ \\
\hline small; $<3 \mathrm{~cm}$ & $18(42)$ \\
\hline \multicolumn{2}{|l|}{ location in SSS } \\
\hline anterior third & $11(25)$ \\
\hline middle third & $19(44)$ \\
\hline posterior third & $13(31)$ \\
\hline \multicolumn{2}{|c|}{ sagittal sinus involvement* } \\
\hline Type I & $22(51)$ \\
\hline Type II-III & $7(16)$ \\
\hline Type IV & $4(9)$ \\
\hline Type V-VI & $10(24)$ \\
\hline \multicolumn{2}{|l|}{ outcome } \\
\hline postop worsening & $2(4.6)$ \\
\hline death & $0(0)$ \\
\hline \multicolumn{2}{|l|}{ extent of resection } \\
\hline Simpson I-II & $37(86)$ \\
\hline Simpson III & $6(14)$ \\
\hline
\end{tabular}

* According to the Sindou classification.

cases the clipped vein was a bridging vein strictly adherent to the tumor. In 1 of 4 patients affected by a middle-third parasagittal meningioma, an MEP alteration was reported within 2 minutes after temporary venous clipping. This information changed the surgical plan, and the vein was not sacrificed. In 3 of 43 cases the ICGVA data were not satisfactory because of the strict relationship between vein and meningioma that required repeated flow assessments focusing on specific vein tracts during the entire resection. In these cases venous flow assessment with a microflow probe was preferred because it was easily repeatable and more precise. Indeed, it provided data on small variations of venous flow values after surgical vein manipulations, thus providing a more accurate venous flow monitoring.

\section{Surgical Outcome}

Data are summarized in Table 1. Overall morbidity was $4.6 \%$ ( 2 of 43 cases). It was related in both cases to surgical manipulation around the motor strip for the re- section of a large middle-third meningioma not occluding the sinus. In both cases a permanent motor deficit associated with a parietal infarction was reported in the postoperative course. Also in both cases, a venous flow modification was not detected on ICGVA at final intraoperative assessment, after tumor resection. No deaths were recorded in our series. A Simpson Grade I-II resection was achieved in 37 patients (86\%) and Grade III in 6 patients (14\%). Simpson Grade I-II was achieved in all 22 patients with Sindou Type I tumors, in 5 of 7 with Type II-III, in 2 of 4 with Type IV, and in 8 of 10 with Type V-VI. A Simpson Grade III resection (with intrasinusal remnant) was achieved in 2 of the 7 patients with Sindou Type II-III; in 2 of the 4 with Type IV; in 2 of the 10 with Type V-VI; in 4 patients with meningiomas located in the middle third of the SSS; and in 2 patients with meningiomas located in the posterior third of the SSS.

\section{Discussion}

\section{An ICGVA-Based Strategy of Vein Management and Tumor Resection}

The ICGVA studies provided useful data for tumor resection at all stages. However, our study shows that ICGVA specifically affected surgical strategy in $20 \%$ of cases. In $16 \%$ of meningiomas completely occluding the SSS (4\% of total cases), ICGVA provided data that diverged from preoperative angiography, changing the resection strategy, and in all cases surgery was uneventful. In 7 cases (16\% of total cases) a vein sacrifice was needed to achieve a radical resection. Venous sacrifice had no postoperative consequences in any of the cases, and was performed according to ICGVA findings alone (42\%) or with the additional assistance of neurophysiological monitoring (58\%). Our data show that should venous sacrifice be needed to improve tumor resection, it can be safely performed with ICGVA and neurophysiological monitoring. In the patients with functional veins, temporary venous clipping under real-time SSEP and MEP monitoring, performed only in those with meningiomas not occluding the sinus, stopped vein sacrifice in $20 \%$ of patients who underwent clipping. Temporary clipping with neurophysiological monitoring was thus predictive of safe venous sacrifice.

However, we must emphasize that there is not universal acceptance of the technique of temporary venous occlusion, or certainly not to the degree that temporary arterial occlusion has been accepted. The effect of venous occlusion, as well as of venous manipulation, can often be delayed and not evident for days after surgery, as occurred in our 2 cases with morbidity. The outcome of the present strategy was a high resection rate with a very low morbidity. Simpson Grade I-II and Grade III rates of resection were achieved in $86 \%$ and $14 \%$ of cases, respectively. In particular, Grade I-II was achieved in $100 \%$ of meningiomas without sinus invasion, in $80 \%$ of meningiomas completely occluding the SSS, and in 63\% of the cases with partial invasion of sinus. The $4.6 \%$ rate of postoperative morbidity with no deaths testifies to the safety of tumor resection performed with such a strategy. 


\section{A. Della Puppa et al.}

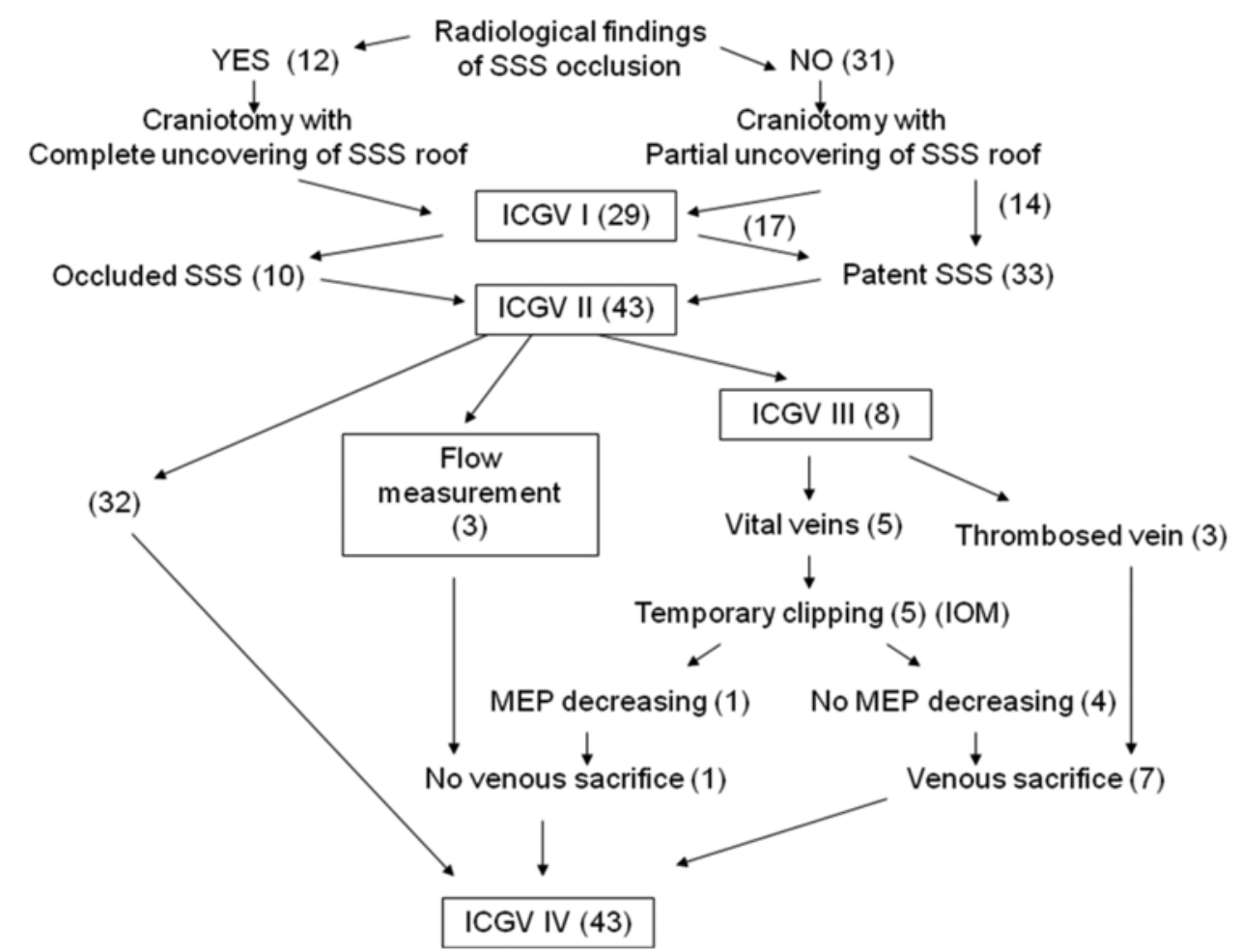

FIG. 5. Algorithm showing surgical strategy and ICGVA (ICGV) data. Values in parentheses denote the number of patients. $\mathrm{IOM}=$ intraoperative neurophysiological monitoring.

\section{Comparison of Our Data With Relevant Literature}

Use of ICGVA in meningioma surgery has already been reported in the literature. ${ }^{2,3}$ So far, the main application of ICGVA in meningioma surgery is before dural opening, to identify and potentially avoid critical draining veins, thus tailoring the approach to the lesion; $3,9,12,24$ in our study this application was not helpful in $15 \%$ of cases. As far as the management of an SSS completely occluded by tumor is concerned, a completely occluded sinus does not guarantee an uneventful selective resection of the SSS with a radical meningioma removal., ${ }^{4} 19$ Sindou and Alvernia ${ }^{19}$ have reported 3 cases of death from brain swelling in patients who underwent complete removal of meningiomas occluding the sinus, without si- nus reconstruction. Tomasello et al. ${ }^{23}$ advocate selective sinus en bloc resection for meningiomas totally occluding the SSS, only when collateral venous outflow anterior to the area of occlusion is proven adequate. We have already reported the role of ICGVA in this subgroup of tumors; ${ }^{2}$ in the present study we report the novel use of ICGVA before dural opening, to detect the cases of sinus nonocclusion that were undetected preoperatively with DSA, safely guiding the resection. This has never been described in the literature. We think that the design of our study does not permit us to make a comparison between intraoperative ICGVA and preoperative DSA sensitivity on this specific issue. However, these data should be taken into consideration for further investigations.
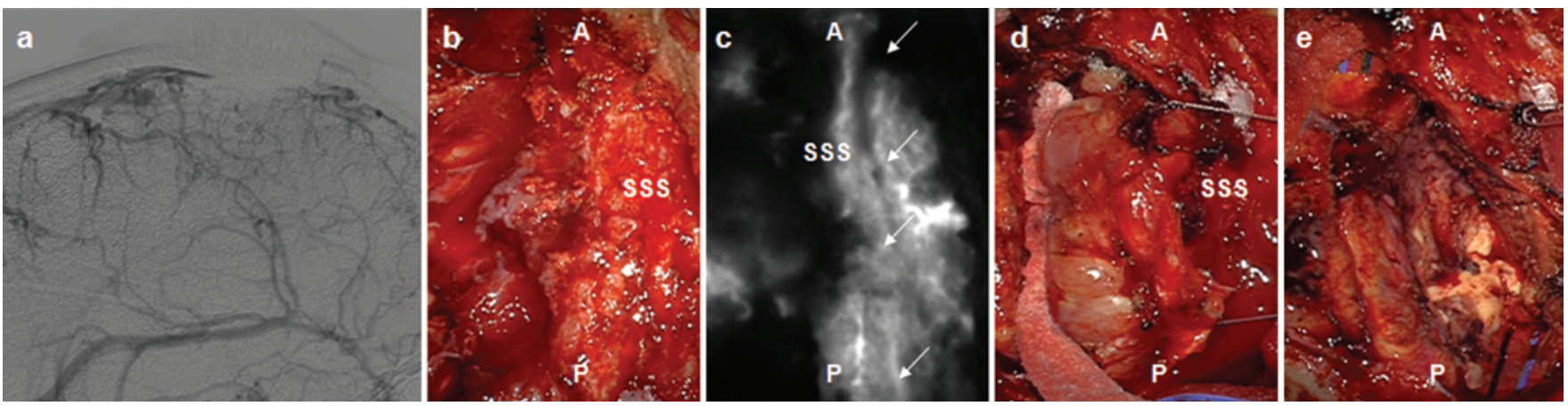

FIG. 6. Preoperative DSA obtained in a 69-year-old woman with parasagittal meningioma of the middle third occluding the SSS (a). b-e: Intraoperative views. The SSS is completely exposed by craniotomy and the meningioma is partially exposed through dural tears (b). An ICGVA study obtained before dural opening (ICGVA I) shows an unexpected filling of SSS (white arrows, c). The SSS is therefore not resected (d) as planned preoperatively, and a Simpson Grade III resection is achieved, leaving an intrasinusal remnant (e). 


\section{The ICGVA-guided resection of parasagittal meningiomas}

It has already been described that intraoperative neurophysiological monitoring of SSEPs and MEPs in the middle-third SSS meningiomas (in proximity to the motor strip) can guide tumor removal. ${ }^{13}$ However, no data have been reported in the literature so far on the combined use of ICGVA and neurophysiological monitoring in meningioma resection. In our experience, intraoperative monitoring was used to monitor SSS ligation and bridging or cortical vein sacrifice (Fig. 4). Temporary venous clipping under ICGVA is a known surgical technique. Ferroli et al. ${ }^{5}$ first described the combination of the venous phase ICGVA with temporary clipping of veins at risk during parasagittal meningioma surgery. In our experience, intraoperative neurophysiological monitoring was accurate in detecting the rare cases of failure of ICGVA findings regarding potential vein sacrifice. Vein sacrifice can therefore be safely performed only by combining temporary clipping and function monitoring. ${ }^{3}$ With regard to the outcome data for our strategy, larger series have reported a radical resection rate of $81 \%-93 \%, 4,14,19,22$ quite similar to the one presented in our study $(86 \%)$. Our data are similar to those published in the literature also when focusing on the pattern of SSS invasion: resection rates of meningiomas invading or not invading the SSS have been reported in $62 \%$ and $96 \%$ of cases, respectively, in the literature, ${ }^{22}$ and in $71 \%$ and $100 \%$, respectively, in our experience. A morbidity rate of $6 \%-11 \%$ and a mortality rate of $0 \%-7 \%$ has been reported by meta-analysis of major series. ${ }^{23}$ In our study, the safety of this strategy seems to be the leading benefit ( $4.6 \%$ morbidity and $0 \%$ mortality).

\section{A Multimodal Strategy for Function Preservation}

In our experience, intraoperative video angiography was an aid to venous management and our tumor resection strategy, improving surgical safety, maintaining the high rates of resection currently reported in the literature. However, our study shows that ICGVA alone may be not sufficient in some selected cases. This consideration arises from the well-known limits of this technique, such as the visualization of superficial vessels, the inaccurate data in cases of high flow related to highly vascularized tumors, the lack of quantitative data to serve as comparison with a baseline during surgery (to have a real-time monitoring of tumor resection), and the complete lack of data predicting brain function. For these reasons, ICGVA ought to be supported by a multimodal strategy. In the 2 patients with morbidity in our study, ICGVA assistance was not able to prevent this occurrence. This concern must be carefully considered in these situations for the possibility of late postsurgical vein thrombosis occurrence not foreseeable at the intraoperative stage.

A direct flow measurement may improve, in selected cases, the significance of ICGVA findings; in our experience, venous flow measurements obtained by Micro Flow Probe provided quantitative flow values and indicated flow direction, especially in cases in which veins were in close relation with the meningioma, and these measures were repeatable without limits, unlike ICGVA. Nevertheless, in our study ICGVA data needed to be complemented by flow measurements in only $7 \%$ of cases. Finally, it should be noted that ICGVA findings on venous flow are not sufficient to predict neurological impairment following vein sacrifice. In our experience, neurophysiological intraoperative monitoring allowed a safe venous sacrifice. Furthermore, functional mapping can identify functional areas and guide surgical manipulation and the operative strategy, particularly in middle-third meningiomas. The high rates of resection with low morbidity that emerged in our experience suggest that ICGVA can be a reliable tool in modern parasagittal meningioma surgery. The design of our study and its results cannot change the current surgical strategy for treating parasagittal meningiomas. However, our study shows that ICGVA, in selected cases and with the assistance of complementary tools, can allow the achievement of high rates of resection with an extremely low morbidity.

\section{Conclusions}

Parasagittal meningiomas deserve dedicated surgical strategies, according to their particular relationship to SSS and parasagittal veins. A surgical strategy focusing on function preservation related to venous management by a multitask approach (ICGVA, functional monitoring, temporary venous clipping, flow measurements) may improve the clinical outcome in these patients.

\section{Disclosure}

The authors report no conflict of interest concerning the materials or methods used in this study or the findings specified in this paper.

Author contributions to the study and manuscript preparation include the following. Conception and design: Della Puppa, Gioffrè, Grandis, Munari. Acquisition of data: Della Puppa, Rustemi, Gioffrè. Analysis and interpretation of data: Della Puppa, Rolma. Drafting the article: Della Puppa, Rustemi. Critically revising the article: Della Puppa, Grandis, Scienza. Reviewed submitted version of manuscript: Della Puppa, Gioffrè, Rolma, Munari, Scienza. Approved the final version of the manuscript on behalf of all authors: Della Puppa.

\section{References}

1. Amin-Hanjani S, Meglio G, Gatto R, Bauer A, Charbel FT: The utility of intraoperative blood flow measurement during aneurysm surgery using an ultrasonic perivascular flow probe. Neurosurgery 58 (4 Suppl 2):ONS-305-ONS-312, 2006

2. d'Avella E, Volpin F, Manara R, Scienza R, Della Puppa A: Indocyanine green videoangiography (ICGV)-guided surgery of parasagittal meningiomas occluding the superior sagittal sinus (SSS). Acta Neurochir (Wien) 155:415-420, 2013

3. Della Puppa A, d'Avella E, Volpin F, Rustemi O, Gioffre' G, Scienza R: Indocyanine green videoangiography (ICGV) in parasagittal meningiomas surgery. Considerations on veins management and brain function preservation. Acta Neurochir (Wien) 155:1475-1476, 2013

4. DiMeco F, Li KW, Casali C, Ciceri E, Giombini S, Filippini $\mathrm{G}$, et al: Meningiomas invading the superior sagittal sinus: surgical experience in 108 cases. Neurosurgery 55:12631274,2004

5. Ferroli P, Nakaji P, Acerbi F, Albanese E, Broggi G: Indocyanine green (ICG) temporary clipping test to assess collateral circulation before venous sacrifice. World Neurosurg 75:122-125, 2011

6. Heros RC: Editorial. Meningiomas involving the sinus. J Neurosurg 105:511-513, 2006 


\section{A. Della Puppa et al.}

7. Heros RC: Editorial. Venous preservation. J Neurosurg 119: 71-72, 2013

8. Jacobs K, Moulin T, Bogousslavsky J, Woimant F, Dehaene I, Tatu L, et al: The stroke syndrome of cortical vein thrombosis. Neurology 47:376-382, 1996

9. Jusué-Torres I, Navarro-Ramírez R, Gallego MP, Chaichana KL, Quiñones-Hinojosa A: Indocyanine green for vessel identification and preservation before dural opening for parasagittal lesions. Neurosurgery 73 Suppl Operative 2:ons145, 2013

10. Keith RA, Granger CV, Hamilton BB, Sherwin FS: The functional independence measure: a new tool for rehabilitation. Adv Clin Rehabil 1:6-18, 1987

11. Kiya K, Satoh H, Mizoue T, Kinoshita Y: Postoperative cortical venous infarction in tumours firmly adherent to the cortex. J Clin Neurosci 8 (1 Suppl 1): 109-113, 2001

12. Nussbaum ES, Defillo A, Nussbaum L: The use of indocyanine green videoangiography to optimize the dural opening for intracranial parasagittal lesions. Neurosurgery 70 (1 Suppl Operative):61-64, 2012

13. Ostrý S, Netuka D, Beneš V: Rolandic area meningioma resection controlled and guided by intraoperative cortical mapping. Acta Neurochir (Wien) 154:843-853, 2012

14. Raza SM, Gallia GL, Brem H, Weingart JD, Long DM, Olivi A: Perioperative and long-term outcomes from the management of parasagittal meningiomas invading the superior sagittal sinus. Neurosurgery 67:885-893, 2010

15. Sakaki T, Kakizaki T, Takeshima T, Miyamoto K, Tsujimoto $\mathrm{S}$ : Importance of prevention of intravenous thrombosis and preservation of the venous collateral flow in bridging vein injury during surgery: an experimental study. Surg Neurol 44:158-162, 1995

16. Simpson D: The recurrence of intracranial meningiomas after surgical treatment. J Neurol Neurosurg Psychiatry 20:2239, 1957

17. Sindou M: Meningiomas invading the sagittal or transverse sinuses, resection with venous reconstruction. J Clin Neurosci 8 (1 Suppl 1):8-11, 2001
18. Sindou M, Auque J: The intracranial venous system as a neurosurgeon's perspective. Adv Tech Stand Neurosurg 26:131216, 2000

19. Sindou MP, Alvernia JE: Results of attempted radical tumor removal and venous repair in 100 consecutive meningiomas involving the major dural sinuses. J Neurosurg 105:514-525, 2006

20. Sughrue ME, Kane AJ, Shangari G, Rutkowski MJ, McDermott MW, Berger MS, et al: The relevance of Simpson Grade I and II resection in modern neurosurgical treatment of World Health Organization Grade I meningiomas. Clinical article. J Neurosurg 113:1029-1035, 2010

21. Sughrue ME, Rutkowski MJ, Shangari G, Fang S, Parsa AT, Berger MS, et al: Incidence, risk factors, and outcome of venous infarction after meningioma surgery in 705 patients. J Clin Neurosci 18:628-632, 2011

22. Sughrue ME, Rutkowski MJ, Shangari G, Parsa AT, Berger MS, McDermott MW: Results with judicious modern neurosurgical management of parasagittal and falcine meningiomas. Clinical article. J Neurosurg 114:731-737, 2011

23. Tomasello F, Conti A, Cardali S, Angileri FF: Venous preservation-guided resection: a changing paradigm in parasagittal meningioma surgery. Clinical article. J Neurosurg 119:7481,2013

24. Ueba T, Abe H, Higashi T, Inoue T: Transdural imaging of meningiomas by indocyanine green videography: the eclipse sign. J Neurol Surg A Cent Eur Neurosurg 74:51-53, 2013

Manuscript submitted August 30, 2013.

Accepted December 10, 2013.

Please include this information when citing this paper: DOI: 10.3171/2013.12.FOCUS13385.

Address correspondence to: Alessandro Della Puppa, M.D., Department of Neurosurgery, Padua University Hospital, Azienda Ospedaliera di Padova, via Giustiniani 2, Padova 35128, Italy. email: alessandro.dellapuppa@sanita.padova.it. 